\title{
ANEURISMA GIGANTE PARASSELAR SIMULANDO TUMOR DE HIPÓFISE
}

\author{
LUIZ AUGUSTO CASULARI ROXO DA MOTTA* - MARIO DE NAZARETH HERMES JR.** \\ MARCO ANTONIO DE ARRUDA FIGUEIREDO** - ANTONIO RICARDO TOLEDO \\ GAGLIARDI*** - LUCILIA DOMINGUES CASULARI DA MOTTA**** - JOSE LUIZ \\ F. DE MENDONCA *
}

\begin{abstract}
RESUMO - Revisamos 7 pacientes com comprometimento da sela turca do raio X do crânio que apresentavam aneurisma gigante da porçāo intracavernosa da artéria carótida (6 casos) e aneurisma da artéria comunicante anterior (1 caso). As alterações encontracias foram: cefaléia (7/7), oftalmoplegia complexa interessando III. IV e VI nervos cranianos (5/7) e ccmpromctimento do $\mathrm{V}$ nervo (4/7), dor ocular (4/7). Outras alteraçōes encontradas foram: sinais meníngeos (2/7), cegueira unilateral (1/7), hemiparesia (1/7) cacosmia (1/7) e quadrantanopsia bitemporal inferior (1/7). Cinco pacientes com aneurisma da porção intracavernosa la artéria carótida beneficiaram-se com a ligadura progressiva da artéria carótida interna a nivel cervical: o outro paciente faleceu antes da realização da operaçāo. o paciente com aneurisma da artéria comunicante anterior foi submetido a clipagem do aneurisma, tendo boa evolução. Baseados weste estudo e em concordância com a literatura, concluímos que o diagnóstico diferencial entre aneurisma localizado na regiāo parasselar e outras patologias dessa área freqüentemente apresenta dificuldade. O diagnóstico definitivo requer a realização de angiografia cerebral. O tratamento cirúrgico, por ligadura da carótida interna na região cervical, è benéfico e quase desprovido de complicações.
\end{abstract}

\section{Giant paraselar aneurysm simulating pituitary tumuur.}

SUMMARY - Seven cases of compromised pituitary fossa at the conventional skull X-ray, who had the final diagnosis of giant aneurysm of the intracavernous portion of the carotid artery $(6$ cases) and one of the anterior communicant artery, are reported. The main findings were: headache (7/7), complex ophtalmoplegia involving the III, IV and VI cranial nerves $(5 / 7)$, compromised $V$ cranial nerve (4/7) and eyeball pain (4/7). Other manitestations were: meningeal signs $(2 / 7)$, unilateral blindness (1/7), hemiparesis (1/7), cacosmia (1/7) and inferior bitemporal quadrantanopsia $(1 / 7)$. Five patients with intracavernous carotid artery aneurysm showed benefits with progressive ucclusion of the internal carotid artery at the cervical level. One died before surgery. The case with anterior communicant artery aneurysm improved after its surgical clipping. Our data, in accord with the literature, support the conclusion that the differential diagnosis of aneurysms in the parasellar egion remains a very difficult task. The accurate final diagnosis requires cerebral angiography and the surgical treatment with progressive occlusion at the cervical portion of the internal carotid artery has a relatively low risk with promising results.

A investigaçāo de massas selares e parasselares apresenta dificuldade devido à grande variedade de lesōes encontradas nessa região e que freqüentemente possuem manifestações clínicas semelhantes $3,4,6,8$. Técnicas recentes, como a tomografia computädorizada (TC) e a ressonância magnética (RM) são efetivas em demonstrar anor-

* Professor Visitante de Endocrinologia da Universidade de Brasilia (UnB), Núcieo de Neuroendocrinologia do Hospital de Base do Distrito Federal (HBDF); ** Residente da Unjdade de Néurocirurgia do HBDF; *:* Professor Assistente de Endocrinologia do HBDF; **.*: Professora Assistente de Ginecologia-Obstetricia da UnB; ***** Médico da Unidade de Radiologia do HBDF; ***** Chefe da Unidade de Neurocirurgia do HBDF. 
malidades intracranianas mas, muitas vezes, as características dessas lesões são inespecificas; 0 diagnóstico depende da localização anatômica e da informação clínica 3,4,6. A RM é particularmente útil em relação à TC, para avaliar ou excluir patologias vasculares, extensão de uma lesão, distorção na anatomia perisselar e massas similarmente hipodensas ao TC 6 . Já a TC é superior à RM em demonstrar calcificação, como nos casos de craniofaringiomas 6 . Infelizmente, grande variedade de lesōes permanece inespecífica, tanto a RM 6 quanto à TC 3,6 . Recentemente em nosso meio, Sampaio 10 chama a atenção sobre a pouca freqüencia com que a TC diagnostica um aneurisma cerebral, exigindo como "prova indispensável, a angiografia cerebral (AC), que mostra não apenas o aneurisma, mas também suas conexões, seu tamanho, espasmos vasculares e condições do colo - elementos essenciais ao tratamento". Quando o aneurisma se localiza em região próxima à sela turca, em particular na porçăo intracavernosa da artéria carótida ( $A C I C)$, pode causar erosão da sua estrutura óssea mimetisando tumor hipofisário $3,4,6-9$ e, se uma cirurgia transesfenoidal é realizada, pode ocasionar efeitos funestos ao paciente 1,5,8. Angiografia carotidea está indicada antes da cirurgia, para demonstrar a anatomia vascular, o seu envolvimento por um tumor, a possibilidade de um aneurisma e a extensão da lesão 3.

O objetivo de nosso trabalho é apresentar a avaliação clínica de pacientes que possuiam alagarmento selar ao raio-X simples do crânio, levando à suspeita diagnóstica de tumor hipofisário e em que se demonstrou aneurisma gigante cerebral pela investigaçāo angiográfica.

\section{CASUISTICA}

Nossa casuística compõe-se de 6 casos de aneurismas gigantes da porçāo cavernosa da artéria carótida interna e um caso de aneurisma da artéria comunicante anterior, atendidos na Unidade de Neurocirurgia do HBDF. O resuma sindrômico desses pacientes encontra-se na tabela 1 .

\begin{tabular}{|c|c|}
\hline Manifestaçāo & Frequência \\
\hline Cefaléia & $7 / 7$ \\
\hline Comprometimento de nervos cranianos: & \\
\hline III, IV e VI & $5 / 7$ \\
\hline $\mathrm{V}$ & $4 / 7$ \\
\hline Dor ocular & $4 / 7$ \\
\hline Sinais meningeos & $2 / 7$ \\
\hline Cegueira unilateral & $1 / 7$ \\
\hline Hemiparesia & $1 / 7$ \\
\hline Cacosmia & $1 / 7$ \\
\hline Quadrantanopsia bitemporal & $1 / 7$ \\
\hline
\end{tabular}

Tabela 1 - Resumo diagnostico de aneurismas giganies com comprometimento selar ( 7 casos).

Caso 1 - EFR, feminino, 36 anos, referia há très meses cefaléia difusa, latejante que se tornou extremamente intensa, localizando-se em região retro-ocular, acompanhada de vômitos. Três dias antes da internaçāo desenvolveu ptose palpebral à esquerda. $\Lambda$ o exame físico apresentava oftalmoplegia complexa à esquerda, interessando III, IV e VI nervos cranianos, associada a comprometimento do $\mathrm{V}$ nervo. $\mathrm{O}$ raio $\mathrm{X}$ do crânio mostrou cesmineralizaçāo do assoalho da sela e da parede lateral externa da órbita. A AC demonstrou aneurisma gigante bocelado na ACIC esquerda, prolongando-se à sela turca e com dilataçăo infundibular em carótida supraclinóide na origem da artéria cerebral média direita.

Caso 2 - MDO, masculino, 20 anos, apresentou 10 dias antes da internação cefaléia súbita, escurecimento da visão e tontura. Apresentava rigidez de nuca e sinaís de Brudzinski e Kernig. A punçāo lombar encontrou-se liquaido céfalo-raquidiano (LCR) hemorrágico. Alargamento selar e calcificaçōes hemisféricas foram os achados ao raio $\mathrm{X}$ do crânio. $\mathbf{A}$ AC mostrou aneurisma gigante da ACIC direita e pequenos aneurismas né artéria cerebral anterior direita.

Caso 3 - JLS, feminino, 67 anos, apresentava história de cefaleia há aproximadamente dois anos, de predominio frontal, acompanhada de dor no globo ocular esquerdo. Associou-se 
dormência na hemiface e hemilíngua esquerda. Tinha antecedentes de hipertensão arterial sistêmica. Ao exame físico encontrou-se oftalmoplegia complexa à esquerda (III. IV e VI nervos), além do acometimento do $V$ nervo também à esquerda. $O$ raio $\mathbf{X}$ de crânio demonstrou erosāo do tubérculo clinóide anterior e discreto alargamento selar. Na AC foi encontrado aneurisma gigante de forma ovalada na ACIC esquerda.

Caso 4 - AMS, feminino, 72 anos, há três meses com cefaléia universal e dor no globo ocular direito, evoluindo para oftalmoplegia à direita três dias após inf́cio do quadro. Permaneceu nesta condiçāo até que a cefaléia e a dor ocular tornaram-se subitamente intensas, ocasião em que procurou nosso serviço. Apresentava oftalmoplegia complexa direita, interessando III, IV e VI nervos cranianos e atingindo também o $V$ nervo do mesmo lado. $O$ raio $x$ de crânio mostrou desmineralização e distensão do dorso selar com aumento do volume da sela turca. A AC mostrou aneurisma gigante da ACIC direita com sinais de trombose.

Caso 5 - MJSA, feminino, 36 anos, informava ter cefaléia fronto-temporal há aproximadamente 13 dias com irradiação para o olho direito $e$, a seguir, apresentou queda da pálpebra direita. O exame físico mostrou oftalmoplegia complexa à direita (III, 1V e VI nervos) associada a acometimento do $\mathrm{V}$ nẹrvo. Erosão do tubérculo clinóide anterior e face lateral do esfenoide, com discreto alargamento selar foram encontrados ao raio $\mathbf{X}$ do crânio. A AC mostrou aneurisma gigante da ACIC direita.

Caso 6 - JSS, masculino, 31 anos, apresentava queixa de cefaléia de pequena intensidade há aproximadamente três meses, que se tornou intensa durante intercurso sexual, melhorando com anelgésicos. Oito dias antes da internaçāo, teve cefaléia súbita e intensa, perdendo a consciência e a forç̣a do lado esquerdo do corpo. Apresentava rigidez de nuca, hemiparesia e sinal de Babinski à esquerda. O rajo $X$ do crânio mostrou grande alargamento selar e à AC foi encontrado grande aneurisma de artéria comunicante anterior que se projetava na regiào selar.

Caso 7 - ASM, feminino, 42 anos, referia diminuiçăo da acuidade visual após ؛clâmpsia há 14 anos e, desde entāo, hipertensăo arterial sistêmica controlada com hidroclorotiazida. Um mês antes da internação passou a apresentar cacosmia e, 12 dias após, cefaléia súbita frontal, pulsátil e de forte intensidade, acompanhada de vômitos. Tinha oftalmoplegia complexa ì direita (III, IV e VI nervos), quadrantanopsia bitemporal inferior e fundos oculares com diminuiçāo bilateral dos pulsos venosos e engurgitamento venoso. $O$ raio $X$ de crânio mostrou alargamento selar (Fig. 1). A CT mostrou grande alargamento selar de

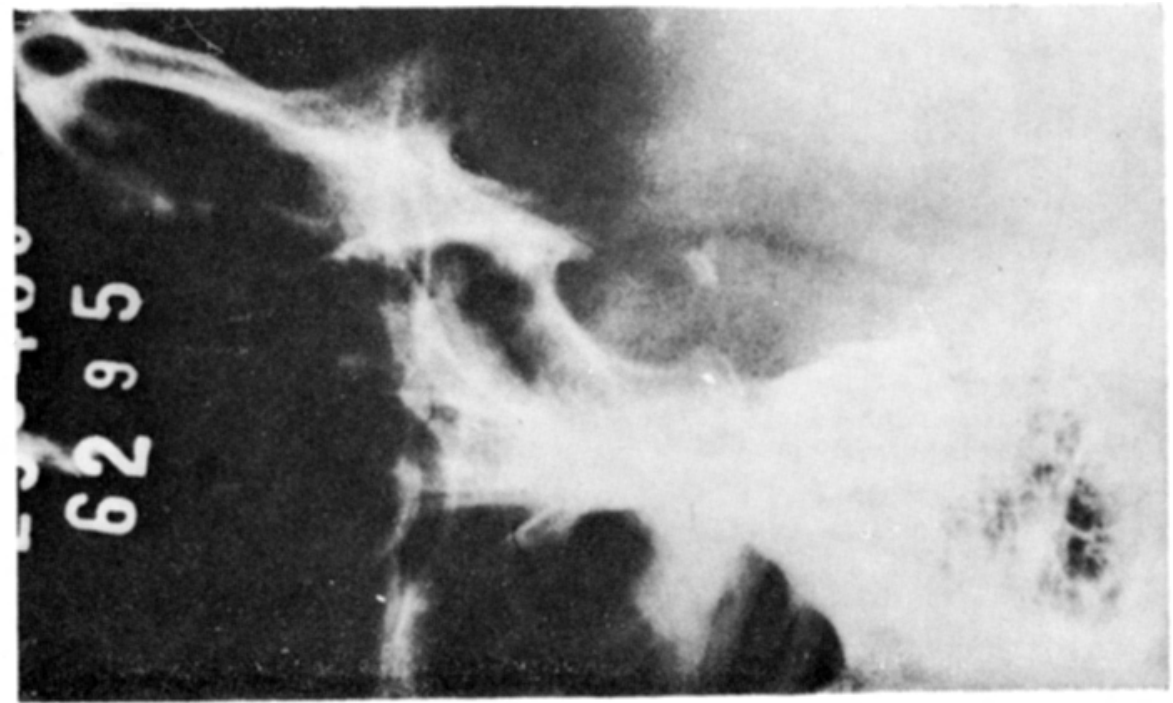

Fig. 1 - Caso \%. Raio-X de cranio mostrando alargamento selar e erosão de clinóide. 
contornos regulares (Fig. 2). A AC evidenciou aneurisma gigante da ACIC dirsita com projeção selar (Fig. 2) e pequeno aneurisma de ACIC esquerda. A pacierte teve piora do seu estado geral, sobrevindo hemorragia digestiva alta por síndrome de Mallory-Weiss, pneumonia e insuficiência respiratória aguda, evoluindo para o óbito.
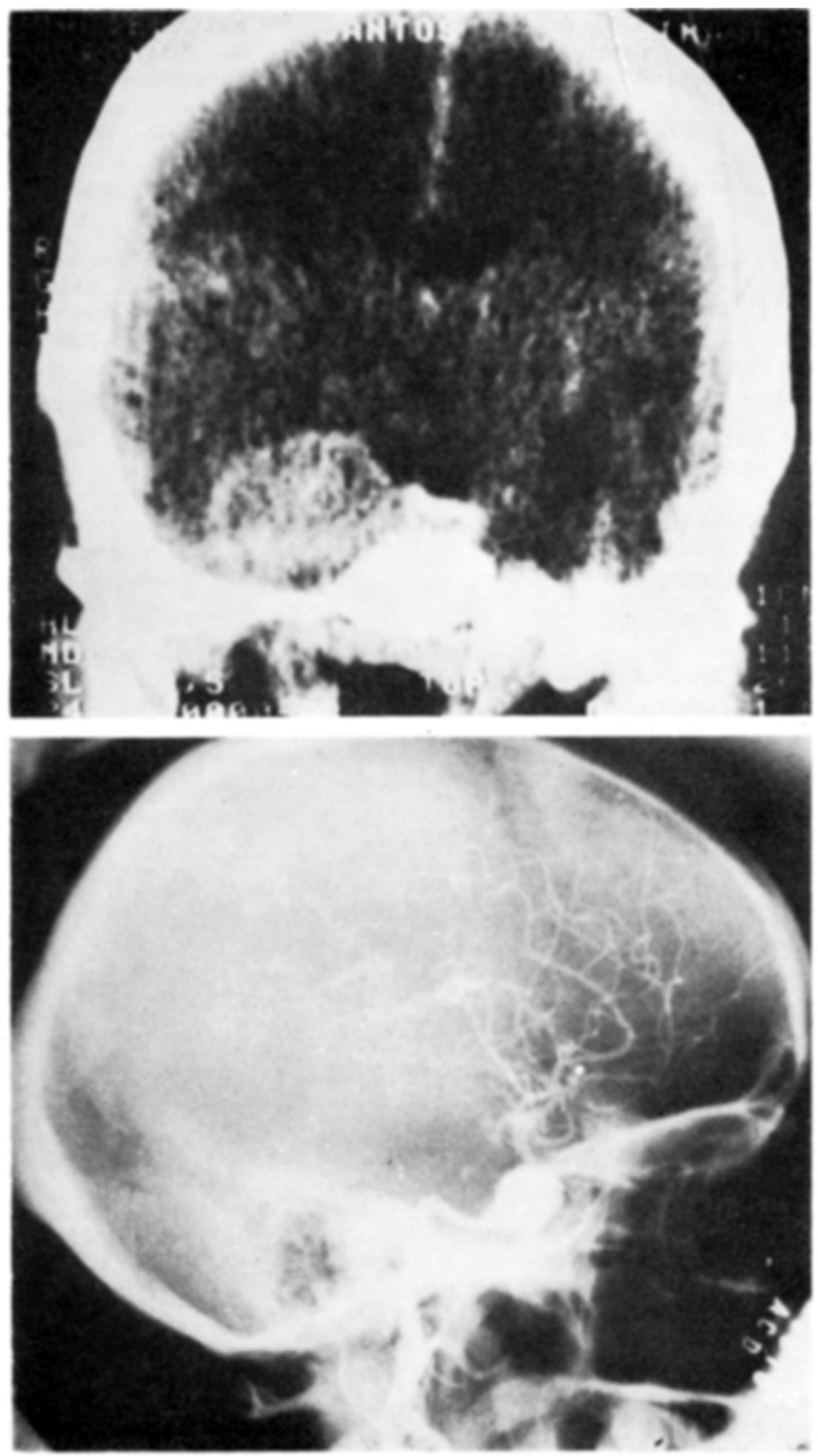

E'ig. 2-Caso \%. No alto, TC do cranio mostrando processo expansivo panasselar. Em baixo, angiografia da carótida direita mostrando aneurisma gigante da artéria carótida cavernosa projetada à região selar. 
Os pacientes de 1 a 5 foram submetidos a ligadura progressiva da artéria carótida interna com clip de Bablock tendo evoluçōes satisfatórias. $O$ caso 6 , com aneurisma da artéria comunicante anterior, foi submetido a clipagem da malformação com clip de Yasargil tendo boa evolução.

\section{COMENTARIOS}

A incidência de aneurismá cerebral varia de 8 a $17 \%$ em autópsias não selecionadas, com predominância entre os 30 e 60 anos, sendo ligeiramente mais freqüente no sexo feminino 10. Com exceção do caso 2, que tinha 20 anos e apresentava dois aneurismas, todos os nossos pacientes tinham idades superiores a 30 anos (média 43 anos), havendo maior incidência no sexo feminino ( 5 casos) que no masculino (2 (asos). Os aneurismas gigantes da ACIC ocorrem quase exclusivamente na mulher entre a quinta e sexta década de vida e, a este respeito, são similares aos aneurismas gigantes supraclinóides 2 . Aneurisma gigante é conceituado como aquele que possui mais de $20 \mathrm{~mm}$ de diâmetro e sua incidência, mimetisando tumor hipofisário necessita ser ainda estabelecida. Naidich e col. 7 encontraram 5 aneurismas do poligono de Willis entre 65 massas parasselares ao examinar retrospectivamente $8500 \mathrm{TC}$. Reich e col. 9 em 1000 TC consecutivas encontraram somente dois aneurismas nesta localizaçāo.

O diagnóstico diferencial das lesões selares e parasselares inclui, além dos adenomas hipofisários e dos aneurismas, outras patologias como meningioma, craniofaringioma, hamartoma, glioma, teratoma e granuloma. Os argumentos diagnósticos para aneurismas intrasselares, segundo Guiot 5 , seriam a ocorrência súbita de defeito visual, com freqüente cegueira unilateral, cefaléia intensa e uma lesão hiperdensa à TC. Daniels e col. 4 acrescentam que à TC um aro de calcificação é mais consistente com aneurisma, enquanto uma calcificação focal sugere craniofaringioma ou meningioma e que os aneurismas são mais excêntricos que outras massas selares. Em nossa casuistica (Tábela 1) a cefaléja, o comprometimento de nervos cranianos unvolvidos na oculomotricidade (III, IV e VI) e na sensibilidade facial (V) e a dor no globo ocular foram as manifestaçōes clinicas mais importantes. Somente dois de nossos pacientes mais recentes foram submetidos à TC e, em ambos, a excentricidade da lesão foi o fator para suspeitar de aneurisma gigante parasselar (Fig. 2).

En todos us nossos pacientes encontramos referência a cefaléia, como piora subita deste sintoma pré-existente ou como aparecimento súbito, geralmente retro-ocular e intensa. Basicamente, sua ocorrência deve-se à hipertensão intracraniana presente em alguns casos e à compressāo de estruturas vizinhas. Este sintoma é freqüentemente associado à ruptura de aneurisma, levando a irritaçāo meníngea por sangue no espaço subaracnóideo, mas somente um dos nossos pacientes (caso 2) apresentou sangue no LCR. A infreqüência de ruptura destes enormes aneurismas, como nos de localização supraclinóide 2 , pode ser devida a uma parede vascular mais espessada ou ao seu apoio a estruturas vizinhas, permitindo maior expansão de volume. Também, similarniente aos aneurismas supraclinóides, pode ocorrer seleção natural, em que um sangramento precoce levaria à morte o paciente ou a uma intervenção cirúrgica, impedindo o desenvolvimento do aneurisma gigante 2 .

A oftalmoplegia complexa, isto $\dot{e}$, o acometimento simultâneo do Ill, IV e VI nervos cranianos, foi freqüente $e$ deve-se ao efeito compressivo da lesão sobre esses nervos, nos seus trajetos através do seio cavernoso e da fenda esfenoidal. A seqüência de comprometimento, a partir do mais próximo da sela turca, é: III, IV, VI e $V$ nervos. Neste ultimo, sua porção oftálmica é a mais freqüentemente envolvida, como encontramos em 4 de nossos pacientes. O comprometimento da oculomotricidade encontrado em nossos casos não é comparável àquele que ocorre em outras lesōes da regiăo suprasselar. A oftalmoplegia complexa foi sempre uniocular e ipsilateral, com instalação rápida do quadro, diferindo da que habitualmente se observa em tumores intrasselares ou parasselares. E possivel que o crescimento do aneurisma seja intermitente ou manos constante que da neoplasia e, assim, a alteraçāo oculomotora pode ocorrer durante um periodo de rápido crescimento ${ }^{2}$. Além do mais os tumores intrasselares crescem na linha média e tendem a ter localização mais posterior 2 . 
A quadrantanopsia bitemporal inferior deve-se ao efeito compressivo do aneurisma gigante a nivel do quiasma óptico. O padrão bitemporal deve-se à compressão das fibras ópticas mediais que se cruzam no quiasma e recebem os estímulos dos campos visuais temporais. Se a compressāo evoluir pode levar a hemianopsia. O fato de se ter apresentado inferiormente deve-se à projeção superior do aneurisma. Caso projetasse para baixo, a quadrantanopsia seria superior. Embora um grande aneurisma não rompido e un tumor possam produzir compressão nas estruturas adjacentes, a campimetria pode não ser suficiente para distingui-los (Walsh 1964, citado por Raymond e Tew ${ }^{8}$ ). Sinais meningeos e perda da consciência com hemiparesia devem-se ao quadro ictal de irritação meningea pelo sangramento, ou no caso da hemiparesia, também ao vasoespasmo e infarto precoce. Em um paciente tivemos referência a cacosmia devida, provavelmente, ao efeito compressivo do aneurisma sobre o uncus do temporal.

Ao raio-X simples do crânio os aneurismas gigantes da carótida cavernosa podem apresentâr os seguintes sinais: erosão do assoalho selar da parede lateral do seio esfenoidal; erosāo da clinóide anterior do lado do aneurisma; erosão com alargamento da fenda esfenoidal; calcificaçāo tipo vascular (ring). Mas, nenhum destes sinais é suficiente para determinar seguramente o diagnóstico de aneurisma. A TC tem grande valor para diagnosticar e demonstrar a extensão de processo parasselar, entretanto, a identificação de anormalidades vasculares dentro da fossa hipofisária e cisterna suprasselar apresenta dificuldade e o diagnóstico diferencial entre um aneurisma parasselar e um tumor permanece problemático ${ }^{3}$. A demonstração de calcificaçóes pelo TC sugere meningioma, craniofaringioma ou aneurisma; geralmente, os adenomas hipofisários nāo as apresentam 4. Recentemente, Cohen e col. 3 desenvolveram método para visualizár as artérias carótidas parasselares com injeçāo endovenosa de contraste e TC com rápida secüência. Em 18 pacientes os segmentos cavernosos das artérias carótidas internas foram bem vistos em 27 dos 28 exames realizados. Assim, preconizam que a TC dinâmica é alternativa viável à angiografia. A RM pode ser mais efetiva do que a $\mathrm{TC}$ em vários estudos de lesōes primárias selares e parasselares. No entanto, muitas anormalidades têm sinais com intensidades semelhantes e sua inespecificidade pode chegar a $70 \%$ dos casos, incluindo entidades comuns, como macroadenomas, gliomas e metástases, e incomuns como hamartomas, germinomas e granulomas sarcóides. O diagnóstico depende grandemente da localizaçāo anatômica e da informação clínica 6 . A arteriografia cerebral é quase sempre diagnóstica, contanto que o saco aneurismático não esteja totalmente trombosado. Ocasionalmente, o tamanho do aneurisma está diminuido devido à trombose reduzir o lúmen visivel à angiografia 8. A angiografia está indicada em massas intrásselares que parecem atípicas de adenoma ou que sāo consistentes com aneurismas ou nıeningiomas 8 .

Com exeção do caso 7, que evoluiu para o óbito antes da cirurgia e que apresentava aneurismas de ambas $\mathrm{AClC}$, todos os nossos pacientes com aneurismas neste local foram tratados com ligadura progressiva da carótida interna a nivel cervical e tiveram boa evolução de sua sintomatologia. Este é o tratamentc de escolha para essa localização de aneurisma, visto que o acesso cirúrgico é difícil no interior do seio cavernoso e o tratamento cirúrgico direto, via intracraniana, apresenta-se perigoso para os aneurismas gigantes 2,8 . O suprimento cerebrovascular passa a ser mantido pela artéria carótida contralateral e pelo sistema vértebro-basilar via polígono de Willis 8. A evacuação do coágulo do saco aneurismático pode ser necessária para descomprimir os nervos ópticos e quiasma particularmente quando o diagnóstico é feito antes de atrofia óptica irreversivel ter ocorridos. 8 .

Em conclusāo, os aneurismas gigantes da ACIC manifestam-se clinicamente por cefaléia e oftalmoplegia complexa súbita, un ocular e ipsilateral, associadas ou não a outros sinais e sintomas. O raio-X do crânio apresenta alteraçóes selares que podem levar ao diagnóstico errôneo de outras patologias dessa região. Os exames radiológicos mais sofisticados (TC e RM) muitas vezes nāo são suficientes para fazer o diagnóstico diferencial das lesões dessa região, sendo necessária complementação com a angiografia cerebral. $O$ tratamento cirúrgico, por ligadura da artéria carótida interna a nível cervical, é benéfico na correção da sintomatologia desses pacientes e apresenta haixo indice de complicaçóes. 


\section{REFERENCIAS}

1. Anderson RD - Tortuosity of the cavernous carotid arteries causing sellar expansion simulating pituitary adenoma. Am J Ruentgenol 126:1203, 1976.

2. Cohen WA, Pinto RS, Kricheff II - Dynamic CT scanning for visualization of the parasellar carotid arteries. Am J Roentgenol 138:905, 1982.

3. Bird AC, Nolan B, Gargano FP. David NJ - Unruptured aneurysm of the supraclinoid carotid artery: a treatable cause of blindness. reurology 20:445, 1970.

4. Daniels DL, Williams AL, Thornton RS, Meyer GA, Cusick JF, Haughton VM - Differential diagnosis of intrasellar tumors by computed tomography. Radiology 141:697, 1981.

5. Guiot $G$ - Considerations on the surgical treatment of pituitary adenomas. In Fahlbusch $R$, von Werder $K$ (eds): Treatment of Pituitary Adenomas. Thieme, Stuttgart. 1978, pg 228 .

6. Karnaze GM, Sartor K. Winthrop JD, Gado MFL, Hodges FJ III - Suprasellar lesions: evaluation with MR imaging. Radiology 161:77, 1986.

7. Naidich TP, Pinto RS, Kushner MJ, Lin JP, Kricheff II, Leeds NE, Chase NE Evaluation of sellar and parasellar masses by computed tomography. Radiolosy 120:91, 1976.

8. Raymond LA, Tew J - Large suprasellar aneurysms imitating pituitary tumour. Neurol Neurosurg Psychiat 41:83, 1978.

9. Reich NE, Zelch JV, Alfidi JR, Meaney TF, Duchesnean PM, Weinstein MA - Tomography computed in the detection of lesions juxtasellar. Radiology 118:333, 1976.

10. Sampaio P - Aneurismas intracranianos: estuđo clinico, Experiêneia com 456 casos clinicos operados. Ars Curandi 124, 1985. 